\title{
Desarrollo de una metodología de enseñanza para el desarrollo humano en psicología de pregrado
}

\section{Development of a teaching methodology for undergraduate human development in psychology}

\author{
Maria A. Rodriguez ${ }^{1 a^{*}}$ \& José M. Espinoza ${ }^{1 \mathrm{~b}}$ \\ ${ }^{1}$ Pontificia Universidad Católica del Perú, Lima, Perú. \\ ${ }^{a}$ Directora del Máster en Psicología Clínica y de la Salud. Obtuvo su PhD en Psicología Clínica y \\ de la Salud en la Universidad de Yeshiva, y completó sus estudios postdoctorales en el Departa- \\ mento de Asuntos para Veteranos. Es psicóloga autorizada en el estado de Nueva York. ${ }^{b}$ Ingeniero \\ informático de la Pontificia Universidad Católica del Perú, con un máster en Ingeniería Informática \\ de la Université de Poitiers, en Francia.
}

Recibido: 01-02-15

Aprobado: 22-05-15

*Correspondencia

Email: marodriguezg@pucp.pe

Notas

\section{Citar Como:}

Rodríguez, M., \& Espinoza, J. (2015). Desarrollo de una metodología de enseñanza para el Desarrollo Humano en psicología de pregrado. Propósitos y Representaciones, 3(1), 71-124. doi: http://dx.doi.org/10.20511/pyr2015.v3n1.69

La implementación de esta metodología de enseñanza se financió con una beca de la Dirección Académica al Profesorado, Pontificia Universidad Católica del Perú. 


\section{Resumen}

Se describe el diseño de una metodología desarrollada para dictar el curso de Desarrollo Humano II, al nivel de pregrado, en una universidad privada de Lima, Perú. El marco teórico incluye la integración de Ciencia Ciudadana y Aprendizaje de Servicio, a través de Tecnologías de la Información y la Comunicación (TIC), incluyendo Wikipedia y el Foro, y trabajo de campo con adultos mayores. Wikipedia, una enciclopedia virtual y gratuita, permite que el alumno cree y edite artículos sobre el contenido del curso. Además, el alumno puede complementar su aprendizaje, distinguir entre información precisa e imprecisa y contribuir al contenido para el público. La metodología tuvo como objetivo que los alumnos aumentaran el pensamiento crítico y la responsabilidad social. Cincuenta y cinco alumnos de una universidad privada de Lima participaron en el curso, 26 alumnos formaron parte del grupo control durante el semestre 2014.1 y 29 alumnos formaron parte del grupo experimental en el semestre 2014.2. Se presentan resultados cualitativos preliminares y la descripción de las fases de planificación e implementación. Análisis del uso del foro sugiere que los alumnos parecen haber utilizado el pensamiento crítico y desarrollado la responsabilidad social hacia el adulto mayor.

Palabras clave: Wikipedia, pedagogía, Desarrollo Humano, educación.

\section{Summary}

The development of a teaching methodology for the undergraduate Psychology course Human Development II in a private university in Lima, Peru is described. The theoretical framework consisted of an integration of Citizen Science and Service Learning, with the application of Information and Communications Technology (ICT), specifically Wikipedia and discussion boards, and fieldwork with Older Adults. Wikipedia, a free digital encyclopedia, allows students to create and edit articles about content within the course. The use of Wikipedia allows students to supplement classroom learning, distinguish between accurate and inaccurate information, and contribute to subject area content online for the public. The teaching methodology aimed to increase critical thinking skills and sense of social responsibility. Fifty-five students of a private university in Lima participated in the course; 26 in the control group during the 2014.1 semester and 29 in the experimental group during the 2014.2 semester. The planning and 
implementation phases of the methodology, as well as initial qualitative findings are discussed. Analysis of discussions in the student forum indicates that students appeared to utilize more critical thinking skills and demonstrated a sense of social responsibility related to the Older Adult.

Keywords: Wikipedia, pedagogy, Human Development, education. 


\section{Introducción}

Las universidades en los lugares más desarrollados del Perú están viviendo el postmodernismo, que se caracteriza por la influencia de la ciencia y la tecnología. El internet provee fuentes adicionales de información para los estudiantes y constituye una potencial herramienta de aprendizaje en la sala de clases. En las salas 2.0; 2009-2011, profesores de educación primaria y secundaria que participaban en un estudio utilizaban tecnología en la sala de clases y más del $90 \%$ obtenía beneficios como la habilidad para acceder a compartiry contextualizar los recursos, la incrementación de la atención y de la motivación de los estudiantes, el aumento de la participación, la participación en actividades colaborativas en grupo y muchas más oportunidades para investigar y desarrollar la creatividad de los estudiantes (Microsoft / DIM$\mathrm{UAB}, 2011)$. Los profesores denotan experiencias positivas tanto para ellos como para los estudiantes, sugiriendo así que el uso del internet en la sala de clases debería integrarse en el programa de enseñanza.

\section{Ciencia ciudadana.}

La ciencia ciudadana implica la participación activa de la gente en distintas fases de la investigación científica, que responde a las necesidades del mundo real (Cohn, 2008). Este enfoque a la investigación está obteniendo cada vez más atención debido al impacto potencial y a las experiencias positivas que las ciencias reportan. Disciplinas tales como la astronomía, la biología, la ecología y la salud humana y del medio ambiente han desarrollado proyectos de investigación que involucran a las personas, y han motivado el desarrollo de una organización en los Estados Unidos para los promotores de la ciencia ciudadana en distintas disciplinas: la Asociación de Ciencia Ciudadana (Citizen Science Association, 2015). En enero del 2015, esta asociación creó una publicación de libre acceso revisada por pares, Citizen Science: Theory and Practice (Ciencia Ciudadana: Teoría y Práctica), para promover el campo.

Las personas se pueden involucrar en las distintas fases del proyecto de investigación: la definición de la pregunta de investigación, la recopilación de información relevante para la conceptualización, el desarrollo de hipótesis, el diseño del estudio, la recopilación de datos, el análisis de muestras y/o datos, la interpretación de los mismos, la obtención de conclusiones, la difusión de los resultados y la formulación de nuevas preguntas (Wiggins \& Crowston, 
2011). Los proyectos de ciencia ciudadana que corresponden a la Educación priorizan la educación de la gente, y su divulgación se realiza ya sea mediante métodos formales o informales. En el estudio de Wiggins y Crowston, todos los proyectos de investigación que utilizan ciencia ciudadana emplearon tecnología para la difusión de los resultados (2011).

Los psicólogos usan cada vez más un enfoque que tiene muchas características similares a la ciencia ciudadana: Investigación de Acción Participatoria (Participatory Action Research, PAR). Este enfoque tiene como objetivo permitir la acción y eliminar las relaciones verticales entre investigadores y participantes; atribuyendo, además, importancia al contexto de un proyecto de investigación (Baum, MacDougall \& Smith, 2006). Esta metodología rechaza el paradigma positivista y, en lugar de ello, se adhiere más al construccionismo social. Este enfoque se basa en el estudio de poblaciones marginales en psicología (Freire, 1972). Por lo general, la comunidad de psicólogos clínicos del Perú utiliza la PAR, y esto es decisivo para probar y adaptar conceptos y teorías desarrollados en los Estados Unidos y Europa al contexto latinoamericano. La fase de difusión de resultados se ve en gran medida influenciada por el deseo de la población. Si la población elige difundir los resultados a otras personas fuera de la comunidad, el investigador puede usar un enfoque de ciencia ciudadana para hacer que los estudiantes ayuden a divulgar los resultados a través de la tecnología o de otros medios identificados.

\section{Aprendizaje en servicio.}

El aprendizaje en servicio es:

una experiencia educacional basada en el curso y con créditos que permite a los estudiantes: (a) participar en una actividad de servicio organizada que cubre necesidades identificadas de la comunidad y (b) reflexionar sobre la actividad de servicio de tal manera que se pueda obtener un mayor entendimiento del contenido del curso, una mayor apreciación de la disciplina y un mayor sentido de la responsabilidad cívica (Bringle \& Hatcher, 1995, p. 112).

Según Howard (1993), el rigor académico no se ve comprometido y se otorgan créditos académicos para la parte de aprendizaje del curso y no para el 
componente de servicio. Además, se debería revaluar el papel educativo de la facultad tradicional. Cook y Kemeny (2014) realizaron un análisis de contenido de entrevistas, con estudiantes que participaron en un programa educativo vacacional de cuatro semanas para veteranos que estaban bajo tratamiento de abuso de sustancias, y encontraron que los estudiantes habían acrecentado su entendimiento del contenido del curso y su autoconciencia. Berrios y sus colegas señalan que los estudiantes perciben el aprendizaje de servicio como una útil estrategia de aprendizaje que les permite integrar el conocimiento, la experiencia profesional y la responsabilidad social, así como resolver problemas complejos (Berrios, Contreras, Herrada, Robles \& Rubio, 2012).

Se utilizó el enfoque de aprendizaje de servicio en un curso de pregrado de introducción a la gerontología en el cual los estudiantes fueron emparejados con personas mayores en una comunidad rural (Dorfman, Murty, Ingram, Evans, Mellor, \& Ivry, 2002). En comparación con estudiantes que no participaron en el componente de aprendizaje de servicio de la clase, los que sí participaron mostraron actitudes más positivas hacia la gente mayor y adquirieron más conocimientos sobre las personas mayores y las comunidades rurales. Del mismo modo, la gente mayor dijo haber tenido una experiencia positiva. Asimismo, Schaber (2015) señaló que los estudiantes aumentaron su conocimiento y confianza al implementar habilidades adquiridas en la sala de clases que implicaban a otros adultos de un servicio diurno para adultos. Al desarrollar habilidades de pensamiento crítico, es posible contribuir a una comunidad (responsabilidad social) a través del aprendizaje de servicio (Sedlak, Doheny, Panthofer \& Anaya, 2003).

Un enfoque combinado de ciencia ciudadana y aprendizaje de servicio en la sala de clases, en el cual los estudiantes difunden conceptos psicológicos a través de Wikipedia y visitan una comunidad de adultos mayores para hacerlos participar en actividades y brindarles información, tiene el potencial de aumentar el sentido de responsabilidad social de los estudiantes, la participación en la experiencia de aprendizaje y las habilidades de pensamiento crítico. Primero se revisa la literatura sobre Tecnologías de la Información y de la Comunicación; y luego, la literatura sobre pensamiento crítico y sentido de responsabilidad social.

\section{Tecnologías de la Información y la Comunicación.}

Las Tecnologías de la Información y la Comunicación (TIC) incluyen las aplicaciones de internet, la tecnología de video, los programas informáticos 
y otras tecnologías que ayudan a usar la información. Se asumen dos cosas respecto a las TIC; primero, que están provocando cambios rápidos y, segundo, que están unificando y estandarizando la cultura (World Youth Report, 2003). Según el Banco Interamericano de Desarrollo (2012), las TIC en América Latina y el Caribe han tenido el mayor crecimiento del mundo. Sin embargo, aún se necesita mucho esfuerzo para que toda la gente tenga el mismo acceso a las TIC. Las TIC permiten a los estudiantes adquirir conocimiento, expresarse de manera ética, resolver problemas y aprender a vivir en paz con otras personas (UNESCO, 2013). Además, las propuestas educacionales para usar TIC deberían considerar los seis aspectos siguientes: la adaptación a las necesidades de los estudiantes, el énfasis en los resultados del aprendizaje, la ampliación del tiempo y el espacio académicos, la creación de nuevas experiencias de aprendizaje, el suministro de experiencias colaborativas y la gestión del uso de conocimiento basado en evidencias para tomar decisiones. Las TIC se pueden integrar con éxito en la sala de clases para crear un enfoque más centrado en el alumno. Se discuten dos formas de TIC: Wikipedia y los foros de discusión.

\section{Wikipedia}

Wikipedia es una enciclopedia digital gratuita que se creó en 2001 y que tiene más de 30 millones de artículos en 287 idiomas (Fundación Wikimedia, 2013). Hay más de un millón de artículos en español (Bienvenidos a Wikipedia, 2015). Cualquiera puede crear o editar artículos sobre temas diversos, así como calificarlos. Wikipedia tiene el potencial para que los académicos compartan información con el público general, pero a muchos artículos les falta el contenido apropiado y las referencias.

La Asociación para la Ciencia de la Psicología (Association for Psychological Science, APS) comenzó una iniciativa en los últimos años para fomentar la difusión de la ciencia psicológica en el mundo usando Wikipedia (Association for Psychological Science, 2014). Más de 2,000 investigadores de psicología y sus estudiantes han editado artículos en Wikipedia en los Estados Unidos. No parece existir un movimiento similar en Latinoamérica. El portal virtual de la APS ayuda a los profesores a asignar el trabajo y difundir artículos a otros mientras del portal para animarlos a comentarlos. A pesar del intento de la iniciativa para alentar la participación de los miembros de la APS y difundir la información al público general, se necesita aún más participación. 
En un análisis de 36 cursos y 640 implicados en la iniciativa, Farzan y Kraut (2013) señalaron que los estudiantes universitarios contribuían a artículos de alta calidad. Los profesores destacaron varias ventajas, tales como: los estudiantes aprenden a escribir sobre temas científicos para el público, aumenta la comprensión del contenido y aumentan sus habilidades de pensamiento crítico. Los estudiantes manifestaron que las tareas los ayudaban a aprender mejor el contenido del curso.

\section{Foros de discusión}

El uso de los foros de discusión está creciendo tanto en el establecimiento universitario como en el campus virtual, como un objeto de aprendizaje. Las plataformas de cursos online de la universidad han ofrecido comúnmente foros de discusión para profesores que se han usado por más de 10 años. Los estudiantes pueden discutir sobre temas fuera de las salas de clases y participar en conversaciones tanto entre sí como con los profesores. En la literatura se citan los siguientes beneficios para los estudiantes: el uso de pensamiento crítico, los estudiantes responden más y son más considerados que en la sala de clases, desarrollan una comunidad de clase más fuerte, es más probable que citen investigaciones y lecturas de clase, y logran un mayor aprendizaje cognitivo y exploratorio (Worcester Polytechnic Institute Academic Technology Center, 2008). Usando la teoría de aprendizaje constructivista, los procesos individuales y grupales implicados en la participación en un foro de discusión deberían crear conocimientos nuevos para los participantes (Harman \& Koohang, 2005). Un foro de discusión brinda a los estudiantes un espacio en el que procesar lo que están aprendiendo y cómo aplicarlo a situaciones del mundo real.

\section{Pensamiento crítico.}

El pensamiento crítico es un proceso en el que buscamos conocimiento a través del razonamiento, de la solución de problemas y de la toma de decisiones, lo que nos permite obtener los resultados deseados eficazmente ( Saiz \& Rivas, 2008). No parece haber una sola definición ampliamente aceptada, sino muchas definiciones, dependiendo de la disciplina. Otras definiciones consideran las habilidades como el ser capaz de separarse de sus propias creencias desde el proceso de evaluación de una discusión (Stanovich $\&$ Stanovich, 2010). Sais y Rivas (2008) argumentan que el pensamiento crítico no debería medirse con preguntas formales con Escalas Likert, 
porque este formato mide más la percepción que el pensamiento. No hay oportunidad para justificar y evaluar las opciones, se mide la comprensión pero no la producción de pensamiento, y los ejemplos que se dan no suelen reflejar situaciones de la vida real, por lo que no interesa mucho quién realiza el examen. Actualmente, al concepto del pensamiento crítico le falta claridad conceptual y metodológica. No se conoce ningún test validado en el ambiente universitario en el Perú; sin embargo, se puede medir el pensamiento crítico a través de cinco habilidades fundamentales del pensamiento: las habilidades de argumentar, formular hipótesis, producir juicios probabilísticos, tomar decisiones y resolver problemas complejos (Saiz \& Rivas, 2008).

Las habilidades de pensamiento crítico son importantes en la vida cotidiana debido a los avances en la tecnología, una mayor abundancia de información disponible, un mundo que cambia rápido, etc. (Halpern, 2003). Más allá de los aspectos prácticos, el pensamiento crítico es esencial para un investigador y practicante, pues les permite hacer conexiones entre observaciones, experiencias pasadas y juicios (Ander-Egg, 2011; Plack \& Greenberg, 2005). Para poder abordar los retos de un país en vías de desarrollo como el Perú, es imperativo que los estudiantes se entrenen en desarrollar habilidades de pensamiento crítico en el ambiente universitario. Disciplinas como la sociología, la filosofía y las ciencias políticas ya han dado pasos hacia la aplicación del pensamiento crítico al contexto latinoamericano y hacia la producción de literatura relevante (Acosta, 2010). Las disciplinas de la psicología y la educación necesitan desarrollar en mayor profundidad este concepto y aplicarlo al contexto latinoamericano.

Varios estudios apoyan el uso del aprendizaje de servicio en la sala de clases para mejorar el pensamiento crítico (Li \& Lal, 2005; Sedlak, Doheny, Panthofer \& Anaya, 2003; Molee, Henry, Sessa \& McKinney-Prupis, 2010; Goldberg \& Coufal, 2009). Mientras tanto, la literatura sugiere que una perspectiva de ciencia ciudadana implique al pensamiento crítico, pero pocos estudios se han realizado para analizar la relación entre ciencia ciudadana y pensamiento crítico. Una tesis doctoral sugiere que el uso de la ciencia ciudadana puede promover diversas habilidades de pensamiento crítico (Basham, 2012). 


\section{Responsabilidad social.}

Muchas universidades latinoamericanas están comenzando a priorizar la responsabilidad social como una característica institucional indispensable. La responsabilidad social universitaria se define como la capacidad de una universidad de comunicar y poner en práctica ciertos principios a través de cuatro procesos clave: la gestión, la enseñanza, la investigación y la extensión (Universidad: Construyendo País, 2004). El concepto de responsabilidad social no tiene antecedentes teóricos concretos; sin embargo, se puede definir como la responsabilidad por los impactos sociales y ambientales que resultan de las decisiones y acciones de organizaciones (Vallaeys, de la Cruz \& Sasia, 2009). Es un esquema ético que supone que uno tiene la obligación de ayudar a la comunidad.

Según la World Declaration on Higher Education for the Twenty-First Century: Visión and Acción (1998) (Declaración Mundial de la Educación Superior para el Siglo XXI: Visión y Acción), los estudiantes de educación superior y el personal universitario deberían "jugar un rol en identificar y abordar los problemas que afectan el bienestar de las comunidades, naciones y de la sociedad global" (UNESCO, 1998, Article 2F). La función de la universidad ya no es suficiente a nivel de la educación, sino que se llama a cada disciplina a poner la teoría en acción. Asimismo, la Conferencia Mundial en Educación Superior señaló que "las instituciones de educación superior en todo el mundo tienen una responsabilidad social de ayudar a cerrar la brecha del desarrollo aumentando la transferencia de conocimiento a través de las fronteras... y trabajando para encontrar soluciones comunes..." (UNESCO, 2009). En un estudio en Chile que medía la percepción de 442 estudiantes de la responsabilidad social universitaria que representa a 11 campos de estudio, más estudiantes participaron como voluntarios en los campos siguientes: Educación Básica, Periodismo, Ingeniería Comercial y Agronomía (Cereceda, 2005). Es aparente que muchas universidades necesitan fomentar a sus estudiantes a participar en proyectos socialmente responsables. Riesch y Potter (2014) señalaron que los científicos que participaron en un proyecto de Ciencia Ciudadana reflexionaron sobre el componente ético de la investigación y cuestionaron la metodología implicada, analizando su rol en la investigación y en la comunidad de estudios. Entretanto, literatura de más de 20 años muestra la influencia positiva del aprendizaje de servicio en la responsabilidad social (Giles Jr. \& Eyler, 1994; Celio, Durlak \& Dymnicki, 2011). 
Desarrollo Humano II consiste en tres horas semanales de teoría con un profesor con nivel de $\mathrm{PhD}$ y dos horas semanales de clases en laboratorio con un instructor con nivel de Máster. El curso cubre desde la adolescencia hasta el fin de la vida, incluyendo aspectos centrales en el desarrollo humano a través del periodo de vida, dificultades psicológicas y sociales que se encuentran en cada etapa de la vida. Además, se hace énfasis en el contexto latinoamericano y, especialmente, el peruano. La sección de laboratorio del curso permite que los estudiantes discutan artículos y películas y conduzcan entrevistas con adolescentes y adultos, trabajando tanto de manera individual como grupal.

La metodología tradicional del curso se cambió al integrar la ciencia ciudadana y el aprendizaje de servicio a través del uso de TIC (i.e. Wikipedia y foros de discusión) y trabajo de campo con adultos mayores. El objetivo de esta nueva metodología era aumentar las habilidades de pensamiento crítico y el sentido de responsabilidad social de los estudiantes (ver figura 1).

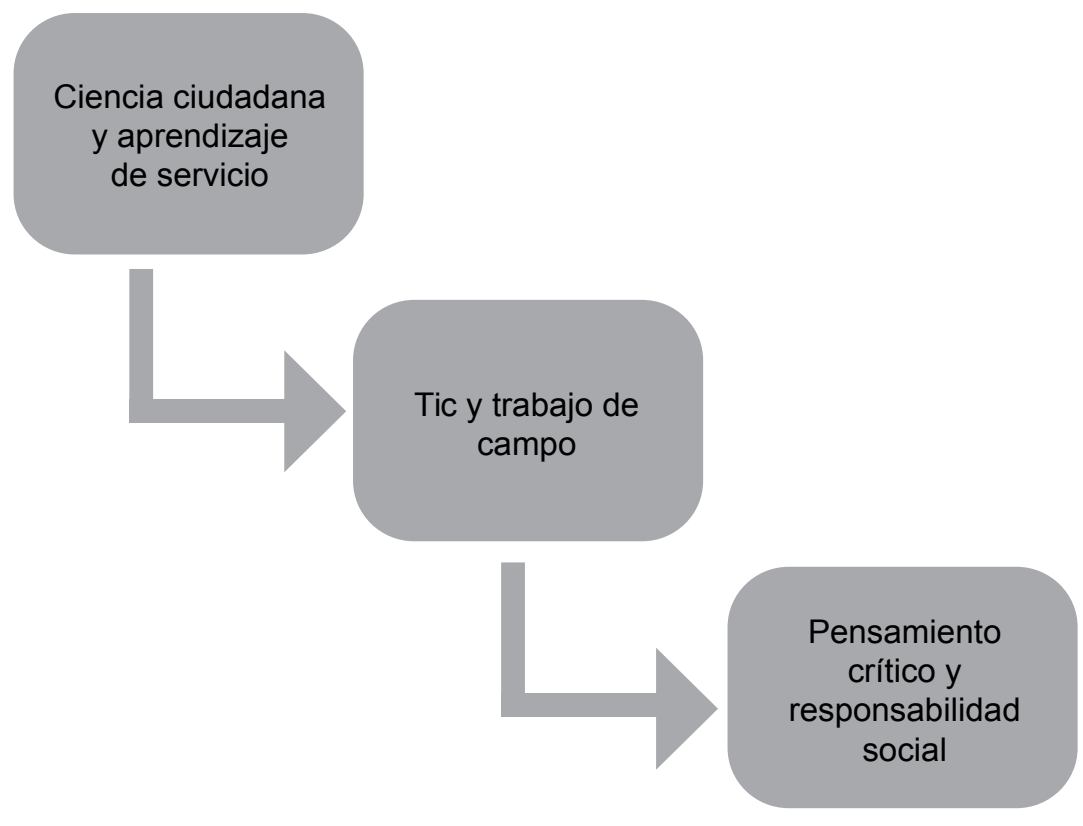

Figura 1. Marco teórico y objetivos. 


\section{Método}

Este proyecto implicó un diseño cuasi experimental.

\section{Participantes.}

El proyecto consistió en 55 estudiantes de Psicología inscritos en Desarrollo Humano II en una universidad privada de Lima, 26 en el grupo de control durante el primer semestre de 2014 y 29 en el grupo experimental en el segundo semestre de 2014. El grupo de control consistía en 21 mujeres y 5 hombres con edades entre los 18 y los 26 años; por otro lado, el grupo experimental consistía en 24 mujeres y 5 hombres con edades entre 18 y 24. Se informó a los estudiantes de los cambios que se estaban llevando a cabo en el curso gracias a una beca de la universidad, pero el consentimiento firmado no era necesario porque el proyecto formaba parte de un proyecto de mejoramiento de calidad y no de investigación. Cuando se administraron encuestas, se aseguró a los estudiantes que eran confidenciales y el profesor no las recolectó individualmente.

\section{Intervención.}

Se dividió a los estudiantes en dos grupos de tres a cinco personas cada uno y se trabajó en dos tareas de Wikipedia. Durante la primera parte de la clase identificaron los temas de interés que correspondían con el contenido de Desarrollo Humano II y que no se encontraban en la versión en español de Wikipedia, y redactaron un artículo. Los estudiantes eran responsables de responder a los comentarios del público de Wikipedia y de cuidar que otra persona no eliminase el artículo. Durante la segunda parte del semestre continuaron perfeccionando el artículo e hicieron que otra gente interactuase con ellos y discutiera el artículo. También se les asignaron artículos que ya existían en Wikipedia, pero que necesitaban información adicional. Asimismo, tenían que participar en discusiones sobre estos artículos. E1 profesor supervisaba todo el trabajo de los estudiantes, y ellos sabían que su participación sería evaluada.

La clase se divide en tres sesiones de laboratorio, cada una con aproximadamente 10 estudiantes. Cada grupo fue a la Casa del Adulto Mayor en otro distrito de Lima, en donde el profesor tenía una relación establecida con un grupo de apoyo para gente con diabetes del tipo 2. Esta 
experiencia tuvo como objetivo reducir los estereotipos sobre los adultos mayores y proporcionar a los estudiantes una experiencia de responsabilidad social, en la cual donaron su tiempo para los adultos mayores. La primera sección de laboratorio facilitaba el diálogo con los miembros del grupo, en grupos más pequeños, discutiendo temas relacionados a cómo se sentían al ser adultos mayores en Lima. También se planeaban actividades dinámicas, como el bingo. En la segunda sección de laboratorio se discutía la resiliencia y se fomentaban las conversaciones que tenían como objetivo ayudar a que los miembros del grupo identificasen formas en las que tienen resiliencia. El último grupo facilitaba actividades grupales, así como un pequeño baile, pues esto era una actividad agradable que se había descubierto en los grupos anteriores. La nota para este componente de Aprendizaje de Servicio se consideraba en la parte de prácticas de la clase.

Para la primera tarea de Wikipedia se tomaron en cuenta para la nota los siguientes criterios: un trabajo sobre la justificación del tema, un trabajo sobre el plan para dividir el trabajo entre los miembros del grupo, la calidad de la participación en el foro, la calidad del artículo, la redacción y la gramática, las referencias (su calidad y su uso apropiado), la incorporación del contexto peruano y que el artículo estuviese publicado aún. Por otro lado, para evaluar la segunda tarea de Wikipedia se utilizaron los siguientes criterios: un trabajo sobre el plan para dividir el trabajo entre los miembros del grupo, la calidad de la participación en el foro, haber participado en la primera tarea de Wikipedia: contribuciones adicionales, referencias agregadas, discusión sobre el artículo; sobre la segunda tarea de edición: discusión sobre el artículo y correcciones completas.

Un tercer requisito para la clase era participar en un foro para discutir las tareas de Wikipedia y compartir posibles prejuicios sobre los adultos mayores, así como proponer ideas para experiencia práctica en la Casa del Adulto Mayor. La función del profesor era facilitar la discusión y considerar la calidad de las respuestas en la nota.

\section{Medidas}

Después de una larga investigación en los libros de psicología para encontrar medidas para el pensamiento crítico y el sentido de responsabilidad social en un contexto hispanoparlante, las medidas fueron adaptadas y creadas. 
El profesor de la sección teórica del curso, tres profesores de la sección de laboratorio y la guía del proyecto (científico informático) trabajaron juntos para desarrollar tales medidas. Como el proyecto empezó en el primer semestre de 2014, después de que la universidad lo financiara, recién se obtuvieron las medidas al final del curso. Primero se creó el autoinforme de fin de semestre, que contenía cuestionarios sobre el uso de la tecnología y Wikipedia, el pensamiento crítico y el sentido de responsabilidad social. Además, se evaluó el pensamiento crítico de las últimas tareas grupales de resumen de artículos usando una rúbrica (ver Anexo A). Las medidas base del grupo experimental fueron similares a las medidas que ya habían sido creadas, pero determinaban las intenciones y experiencias futuras en otros cursos de psicología con pensamiento crítico y un sentido de responsabilidad social. Por otro lado, las medidas al fin de semestre fueron idénticas, con la adición de un pequeño cuestionario online que evaluaba las percepciones de los estudiantes sobre haber completado efectivamente las tareas de Wikipedia. La figura 2 describe el diseño de este proyecto.

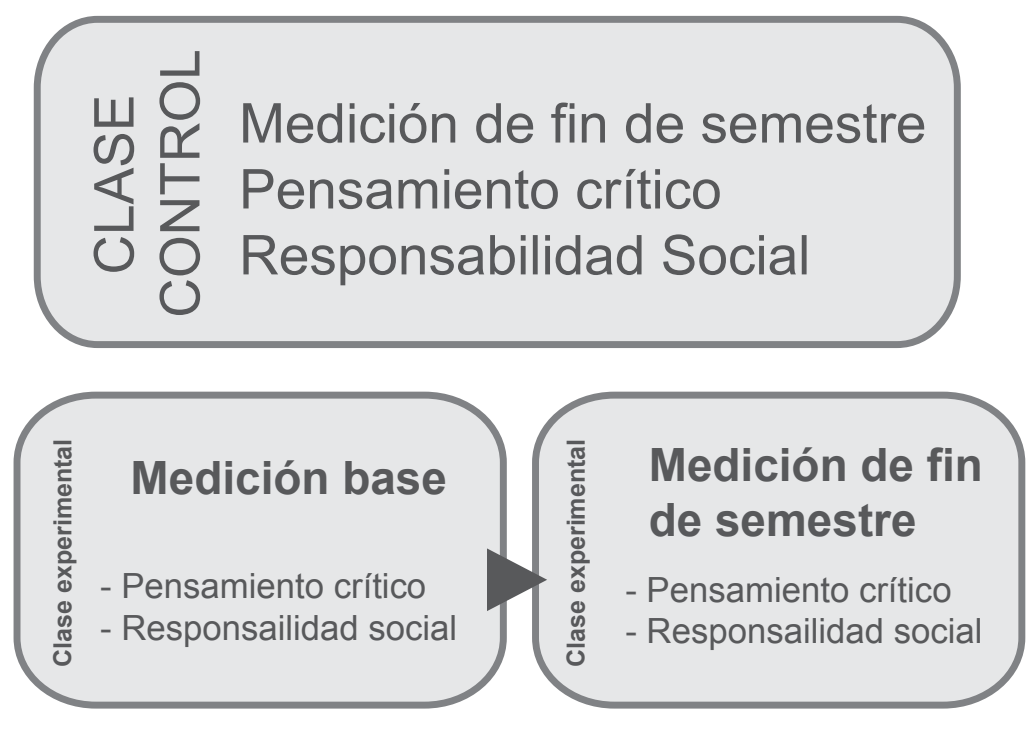

Figura 2. Diseño del proyecto. 


\section{Pensamiento crítico.}

Se midió el pensamiento crítico a través de un autoinforme y de una evaluación de las críticas de los artículos grupales llevada a cabo por los instructores de laboratorio (ver anexos A y B). Los tres instructores de laboratorio y el profesor crearon cuestiones que se adherían a los cinco ámbitos del pensamiento crítico: la habilidad de argumentar, de formular hipótesis, de producir juicios probabilísticos, de tomar decisiones y de resolver problemas complejos (Saiz \& Rivas, 2008). Las preguntas del autoinforme que se utilizaron para el grupo experimental hicieron que los estudiantes evaluaran sus experiencias en cursos de psicología anteriores y sus expectativas para el curso de Desarrollo Humano II en una escala de cinco puntos que iba desde el acuerdo total hasta el desacuerdo total en el desarrollo de cada ámbito. Por otro lado, al final de los dos cursos, los estudiantes calificaban los ejercicios de laboratorio de análisis de entrevistas, trabajo en grupo, discusión de textos y lecturas críticas en una escala de cinco puntos que iba desde el acuerdo total hasta el desacuerdo total. La evaluación de las críticas del artículo se llevó a cabo usando una rúbrica del uno al cuatro para los cinco ámbitos, en la que el uno indicaba la falta de ámbito y la cinco un nivel óptimo del ámbito.

\section{Responsabilidad social.}

Se conceptualizó la responsabilidad social usando la experiencia en Chile, Universidad: Construyendo País (2004). Al inicio del estudio se preguntó al grupo experimental sobre su percepción de cursos previos y su expectativa para el curso actual, relacionada con cómo estos permiten a los estudiantes reconocer la importancia de la responsabilidad social y las capacidades que poseen y cómo estas pueden ser útiles para la responsabilidad social y motivar a los estudiantes a participar en proyectos sobre responsabilidad social. También se preguntó a los estudiantes si habían participado en proyectos de responsabilidad social anteriormente, o si planeaban hacerlo durante ese semestre o en el futuro. La evaluación durante la última clase para los dos cursos preguntaba si el curso había ayudado a los estudiantes a desarrollar las tres habilidades previamente mencionadas. De la misma manera, se usó la escala del uno al cinco, que va desde el acuerdo total hasta el desacuerdo total. 


\section{Análisis.}

Se usará SPSS versión 21 para analizar las diferencias en pensamiento crítico y responsabilidad social entre los dos grupos durante la última clase, así como las diferencias antes y después de la metodología que se hubiese aplicado al grupo experimental. El análisis actual de este estudio se basa en los descubrimientos preliminares del uso del cuestionario sobre tecnología y el análisis de contenido de los comentarios de los foros.

\section{Resultados}

Los estudiantes de los dos grupos parecen similares de manera global. Los estudiantes utilizan, principalmente, laptops y smartphones y suelen acceder a Facebook y a la intranet para la universidad. Se comunican esencialmente por mensajes de texto, usando el Messenger de Facebook, emails y llamadas por celular. Los estudiantes usan Facebook en promedio, como máximo, mensualmente, y buscan contenidos generales. Consideran que no es fiable dada la facilidad con la que uno puede modificar el contenido. Prefieren actividades de aprendizaje práctico, tanto dentro como fuera de la sala de clases. Según el autoinforme, el grupo experimental percibió que las clases anteriores de psicología les ayudaron a desarrollar habilidades de pensamiento crítico. También consideraron que las clases de psicología los motivaron a participar en la responsabilidad social, pero no estaban, en su mayoría, implicados en proyectos relacionados con ella. Los estudiantes tenían expectativas similares respecto a que el curso de Desarrollo Humano II aumentaría su sentido de responsabilidad social.

Las conversaciones en un foro sobre la creación de un artículo se relacionaban principalmente con preguntar la opinión de otros estudiantes para evitar que el artículo fuese eliminado, dando consejos basados en su interacción con la comunidad de Wikipedia a sus compañeros de clase y haciendo preguntas concretas sobre las tareas. Señalaron que se sintieron frustrados durante la creación de una nueva tarea porque la mayoría de los artículos de los demás grupos fueron eliminados de Wikipedia varias veces y no pudieron obtener respuestas concretas de la comunidad de Wikipedia sobre cómo mejorar su artículo. Muchos estudiantes lucharon con la incapacidad de usar fuentes primarias, pues Wikipedia no aconseja su uso. Miembros de las comunidades de otras disciplinas, como la Física, comentaron en artículos que no pertenecían a sus áreas de trabajo. Hubo factores más allá 
del control del profesor que hicieron que los estudiantes tuvieran que usar habilidades de pensamiento crítico para crear artículos aceptables para Wikipedia. Por otro lado, conversaciones sobre la edición de un artículo y la mejora del original creaban y estimulaban reflexiones sobre el contenido del artículo. Los estudiantes describieron la tarea como más fácil y se sentían más tranquilos y felices con esta tarea debido al entendimiento mejorado de cómo participar eficientemente en Wikipedia.

El foro estimuló más debates sobre la parte de aprendizaje de servicio del curso. Los estudiantes demostraron un sentido de cohesión, compartiendo experiencias en la Casa del Adulto Mayor y dando recomendaciones a otros grupos. Además, demostraron la habilidad de reflexionar sobre sus percepciones originales sobre los adultos mayores (por ejemplo, "están deprimidos y no les gusta mucha actividad"), y comentaron los cambios en su percepción debido a la interacción con adultos mayores. Basándose en conversaciones con sus abuelos y leyendo fuentes externas a la clase, comenzaron a proponer actividades que pudieran implementar. Después de haber participado en la actividad de aprendizaje de servicio, muchos estudiantes reflexionaron sobre cuán activos, motivados y felices parecían los adultos mayores. Un estudiante comentó:

Creo que fue una experiencia encantadora porque esta gente quiere que la escuchen, y eso es lo que pudimos hacer cuando los visitamos. Aunque no pudimos completar todas las actividades que planeamos, nos contaron sobre sus sentimientos y experiencias, y surgieron temas que no pensé fueran muy comunes, como cuánto les gusta bailar y viajar. Pensaba que hablar sobre los sentimientos de envejecer y de salud sería difícil debido a su edad y a las enfermedades que tienen (como diabetes), pero respondieron a todas las preguntas de manera abierta y con entusiasmo.

Los estudiantes mostraron habilidades de pensamiento crítico al debatir qué actividades deberían implementarse en el componente de aprendizaje de servicio y al comentar sobre las opiniones de otros. También analizaron las probabilidades de que su trabajo fuese aceptado o rechazado por la comunidad basándose en sus experiencias pasadas y en conversaciones con la comunidad de Wikipedia y ayudando a otros estudiantes que habían tenido dificultades con la escritura de su artículo. También demostraron un sentido 
de responsabilidad social relacionado con el componente de aprendizaje de servicio, en el cual dieron recomendaciones a futuros estudiantes y pensaron sobre la importancia de su papel al interactuar con adultos mayores.

\section{Discusión}

El uso de la ciencia ciudadana y del aprendizaje de servicio tiene el potencial de aumentar las habilidades de pensamiento crítico de los estudiantes y su sentido de responsabilidad social. La literatura sugiere una relación entre estos ámbitos y resultados, pero hay retos que miden los objetivos en el ambiente educacional en América Latina. Primero, esta metodología se desarrolló en el contexto de una clase y se consideró la carga del estudiante. Se crearon medidas simples que pudieran informar el refinamiento de la metodología de enseñanza. No se validaron medidas porque el proyecto estaba conceptualizado como un proyecto de mejora de calidad, y no como una investigación. Estudios futuros deberían validar estas medidas e intentar aplicar los descubrimientos a un público más amplio en otras disciplinas. Además, existe ambigüedad en la comunidad científica sobre cómo definir estos conceptos, medirlos y, además, hacerlo en español.

Los psicólogos comparten un sentido de responsabilidad social para difundir información exacta al público. Existen muchos estereotipos sobre los adultos mayores, gente vulnerable y a menudo ignorada en América Latina. Los estereotipos negativos ponen énfasis en el deterioro físico y mental, mientras que los estereotipos positivos describen una edad dorada sin preocupaciones; ninguno de estos estereotipos es bueno para los adultos mayores (Yuste, Rubio \& Aleixandre, 2004). Esta experiencia de aprendizaje pareció aumentar la sensibilidad al adulto mayor y reforzar el material aprendido en la sala de clases.

Un artículo futuro discutirá las variables de resultados, es decir, el pensamiento crítico y el sentido de responsabilidad social, y dará sugerencias para medirlos en el futuro. Se están haciendo varios cambios en el curso de manera simultánea, y es difícil separar los efectos de cada variable puesto que tienen cosas en común. Toda la Gestalt de la experiencia debería considerarse. Descubrimientos preliminares sugieren que los estudiantes se pueden beneficiar de la metodología que propone el curso de Desarrollo Humano II a nivel de pregrado. 


\section{Referencias}

Acosta, Y. (2010). Pensamiento crítico, sujeto y democracia en América Latina. Utopia y Praxis Latinoamericana, 15(50), 15-43.

Ander-Egg, E. (2011). Aprender a Investigar. Córdoba: Brujas.

Association for Psychological Science. (2014). APS Wikipedia Initiative. Retrieved from http://www.psychologicalscience.org/index.php/ members/aps-wikipedia-initiative

Atkinson, M.P. \& Hunt, A.N. (2008). Inquiry-Guided Learning in Sociology. Teaching Sociology, 36(1), 1-7.

Banco Interamericano de Desarrollo, BID (2012). Construyendo puentes, Creando oportunidades: La banda ancha como catalizador del desarrollo económico y social en los países de América Latina y el Caribe.

Basham, M. (2012). Pathways of Knowing: Integrating Citizen Science and Critical Thinking in the Adult ELL Classroom (Doctoral Dissertation). Retrieved from Arizona State University Respository.

Baum, F., MacDougall, C. \& Smith, D. (2006). Participatory Action Research. J. Epidemiol Community Health, 60 (10), 854-857.

Berríos, V., Contreras, M., Herrada, M., Robles, M. \& Rubio, X. (2012). Resultados de Aprendizaje Servicio en la UC: Informe final de investigación. Santiago, Programa Aprendizaje Servicio, Pontificia Universidad Católica de Chile.

Bienvenidos a Wikipedia (January 14, 2015). In Wikipedia. Retrieved January 13, 2015, from https://es.wikipedia.org/wiki/Wikipedia: Portada.

Bringle, R. \& Hatcher, J. (1995). A service-learning curriculum for faculty. Michigan Journal of Community Service Learning, 2, 112-122.

Celio, C.I., Durlak, J. \& Dymnicki, A. (2011). A Meta-analysis of the impact of service-learning on students. Journal of Experiential Education, 34(2), 164-181.

Cereceda, L. (2005). Percepción de los estudiantes en torno a la Responsabilidad Social Universitaria en la Pontificia Universidad Católica de Chile. Proyecto Universidad Construye País. Retrieved from http://vidauniversitaria.uc.cl/proyectos/documentos/documentos/rsu.pdf 
Citizen Science Association (2015). Overview. Retrieved January 30, 2015; from https://www.library.cornell.edu/research/citation/apa.

Cohn, J.P. (2008). Citizen Science: Can Volunteers Do Real Research? BioScience, 58(3), 192-197.

Cooke, C.A. \& Kemeny, E.M. (2014). Student Perspectives on the Impact of Service Learning on the Educational Experience. Schole: A Journal of Leisure Studies \& Recreation Education, 29(1), 102-111.

Dorfman, L.T., Murty, S., Ingram, J.G., Evans, R.J., Mellor, J.M. \& Ivry, J. (2002). Incorporating Intergenerational Service-Learning into an Introductory Gerentology Course. Advancing Gerentological Social Work Education, 39(1/2), 219-240.

Farzan, R. \& Kraut, R. (2013). Wikipedia classroom experiment: bidirectional benefits of students'engagement in online production communities. In: SIGCHI Conference on Human Factors in Computing Systems, 27 April - 2 May 2013, Paris.

Freire P. (1972). Pedagogy of the oppressed. Harmondsworth: Penguin.

Giles Jr., D.E. \& Eyler, J. (1994). The impact of college community service laboratory on students' personal, social, and cognitive outcomes. Journal of Adolescence, 17(4), 327-339.

Goldberg, L.R. \& Coufal, K.L. (2009). Reflections On Service-Learning, Critical Thinking, and Cultural Competence. Journal of College Teaching \& Learning, 6(6), 39-50.

Halpern, D.F. (2003). Thought and knowledge: an introduction to critical thinking. New Jersey: Lawrence Erlbaum Associates, Inc.

Harman, K. \& Koohang, A. (2005). Discussion Board: A Learning Object. Interdisciplinary Journal of Knowledge and Learning Objects, 1, 67-77.

Howard, J. (Ed.). (1993). Praxis I: A faculty casebook on community service learning. Ann Arbor, MI: Office of Community Service Learning Press, University of Michigan.

Li, X. \& Lal, S. (2005). Critical reflective thinking through service-learning in multicultural teacher education. Intercultural Education, 16(3), 217234. 
Microsoft / DIM-UAB (2011). Investigación: Aulas 2.0 2009-2011, Memoria - Julio 2011. Pangea. Recovered from http://peremarques.pangea.org/ aulas20/

Molee, L.M., Henry, M.E., Sessa, V.I. \& McKinney-Prupis, E.R. (2010). Assessing Learning in Service-Learning Courses through Critical Reflection. Journal of Experiential Education, 33(3), 239-257.

Plack, M. \& Greenberg, L. (2005). The Reflective Practitioner: Reaching for Excellence in Practice. Pediatrics, 116 (6), 1546-1552. doi: 10.1542/ peds.2005-0209

Riesch, H. \& Potter, C. (2014). Citizen science as seen by scientists: Methodological, epistemological and ethical dimensions. Public Understanding of Science, 23(1), 107-112.

Saiz, C. \& Rivas, S.F. (2008). Evaluación en pensamiento crítica: una propuesta para diferenciar formas de pensar. Ergo, Nueva Época, 22-23, 25-66.

Schaber, P. (2010). Teaching Program Development and Evaluation Through a Service Learning Project in Community-Based Adult Day Services. Occupational Therapy in Health Care, 24(1), 107.

Sedlak, C.A., Doheny, M.O., Panthofer, N. \& Anaya, E. (2003). Critical Thinking in Students' Service-Learning Experiences. College Teaching, 51(3), 99-104.

Stanovich, K.E. \& Stanovich, P.J. (2010). A framework for critical thinking, rational thinking, and intelligence. In D. Preiss \& R.J. Sternberg (Eds.), Innovations educational psychology: Perspectives on learning, teaching and human development (pp. 195-237). New York: Springer.

UNESCO (1998). World Declaration on Higher Education for the TwentyFirst Century: Vision and Action. World Conference on Higher Education. Retrieved from http://www.unesco.org/education/educprog/ wche/declaration_eng.htm

UNESCO (2009). 2009 World Conference on Higher Education: The New Dynamics of Higher Education and Research For Societal Change and Development. Paris. Retrieved from http://www.unesco.org/ fileadmin/MULTIMEDIA/HQ/ED/ED/pdf/WCHE_2009/FINAL\%20 COMMUNIQUE\%20WCHE\%202009.pdf 
UNESCO (2013). Enfoques Estratégicos Sobre las TICS en Educación en América Latina y el Caribe. Retrieved January 30, 2015 from http:// www.unesco.org/new/fileadmin/MULTIMEDIA/FIELD/Santiago/ images/ticsesp.pdf

Universidad: Construyendo País (2004). Observando la Responsabilidad Social. Recovered from www.construyepais.cl

Vallaeys, F., De la Cruz, C. \& Sasia, P. (2009). Responsabilidad Social Universitaria, Manual de primeros pasos. México, McGraw-Hill Interamericana Editores, Inter-American Development Bank.

Wiggins, A. \& Crowston, K. (2011). From Conservation to Crowdsourcing: A Typology of Citizen Science. In Proceedings of the 44th Annual Hawaii International Conference on System Sciences. Koloa, HI, 4-7 January, 2011.

Wikimedia Foundation. Ten years of sharing and learning, 2013. Retrieved from https://wikimediafoundation.org/wiki/Annual_Report

Worcester Polytechnic Institute Academic Technology Center (2008). Benefits of Using Discussion Boards in Your Classes. Retrieved January 30, 2015 from https://www.wpi.edu/Academics/ATC/Collaboratory/ Idea/boardbenefits.html

World Youth Report (2003). Youth and Information and Communication Technologies (ICT). Retrieved January 30, 2015, fromhttp://www. un.org/esa/socdev/unyin/documents/ch12.pdf

Yuste, N., Rubio, R. \& Aleixandre, M. (2004). Introducción a la Psicogerontología. Madrid: Ediciones Pirámide. 


\section{Anexo A}

Encuesta de Medición de Pensamiento Crítico y Sentido de la Responsabilidad Social

\section{Parte I: EI Pensamiento crítico}

Marque el grado de acuerdo frente a las siguientes frases con respecto a los ejercicios de análisis de las entrevistas

\begin{tabular}{|l|l|l|l|l|l|}
\hline & $\begin{array}{c}\text { Muy de } \\
\text { acuerdo }\end{array}$ & $\begin{array}{c}\text { De } \\
\text { acuerdo }\end{array}$ & $\begin{array}{c}\text { Ni de } \\
\text { acuerdo ni en } \\
\text { desacuerdo }\end{array}$ & $\begin{array}{c}\text { En } \\
\text { desacuerdo }\end{array}$ & $\begin{array}{c}\text { Muy en } \\
\text { desacuerdo }\end{array}$ \\
\hline $\begin{array}{l}\text { Han desarrollado mi } \\
\text { capacidad para argumentar. }\end{array}$ & & & & & \\
\hline $\begin{array}{l}\text { Han desarrollado mi } \\
\text { capacidad para plantear } \\
\text { hipótesis. }\end{array}$ & & & & & \\
\hline $\begin{array}{l}\text { Han desarrollado mi } \\
\text { capacidad para emitir } \\
\text { juicios de probabilidad. }\end{array}$ & & & & & \\
\hline $\begin{array}{l}\text { Han desarrollado mi } \\
\text { capacidad de decidir. }\end{array}$ & & & & & \\
\hline $\begin{array}{l}\text { Han desarrollado mi } \\
\text { capacidad para resolver } \\
\text { problemas complejos. }\end{array}$ & & & & & \\
\hline
\end{tabular}

Marque el grado de acuerdo frente a las siguientes frases con respecto a la experiencia de trabajos en grupo.

\begin{tabular}{|l|l|l|l|l|l|}
\hline & $\begin{array}{c}\text { Muy de } \\
\text { acuerdo }\end{array}$ & $\begin{array}{c}\text { De } \\
\text { acuerdo }\end{array}$ & $\begin{array}{c}\text { Ni de } \\
\text { acuerdo ni en } \\
\text { desacuerdo }\end{array}$ & $\begin{array}{c}\text { En } \\
\text { desacuerdo }\end{array}$ & $\begin{array}{c}\text { Muy en } \\
\text { desacuerdo }\end{array}$ \\
\hline $\begin{array}{l}\text { Han desarrollado } \\
\text { mi capacidad para } \\
\text { argumentar. }\end{array}$ & & & & & \\
\hline $\begin{array}{l}\text { Han desarrollado mi } \\
\text { capacidad para plantear } \\
\text { hipótesis. }\end{array}$ & & & & & \\
\hline $\begin{array}{l}\text { Han desarrollado mi } \\
\text { capacidad para emitir } \\
\text { juicios de probabilidad. }\end{array}$ & & & & & \\
\hline $\begin{array}{l}\text { Han desarrollado mi } \\
\text { capacidad de decidir. }\end{array}$ & & & & & \\
\hline $\begin{array}{l}\text { Han desarrollado mi } \\
\text { capacidad para resolver } \\
\text { problemas complejos. }\end{array}$ & & & & & \\
\hline
\end{tabular}


Marque el grado de acuerdo frente a las siguientes frases con respecto a $\underline{\mathbf{l a}}$ experiencia de discusión de textos en clase

\begin{tabular}{|c|c|c|c|c|c|}
\hline & $\begin{array}{l}\text { Muy de } \\
\text { acuerdo }\end{array}$ & De acuerdo & $\begin{array}{l}\text { Ni de acuerdo ni } \\
\text { en desacuerdo }\end{array}$ & $\begin{array}{c}\text { En } \\
\text { desacuerdo }\end{array}$ & $\begin{array}{c}\text { Muy en } \\
\text { desacuerdo }\end{array}$ \\
\hline $\begin{array}{l}\text { Han desarrollado } \\
\text { mi capacidad para } \\
\text { argumentar. }\end{array}$ & & & & & \\
\hline $\begin{array}{l}\text { Han desarrollado } \\
\text { mi capacidad para } \\
\text { plantear hipótesis. }\end{array}$ & & & & & \\
\hline $\begin{array}{l}\text { Han desarrollado } \\
\text { mi capacidad para } \\
\text { emitir juicios de } \\
\text { probabilidad. }\end{array}$ & & & & & \\
\hline $\begin{array}{l}\text { Han desarrollado mi } \\
\text { capacidad de decidir. }\end{array}$ & & & & & \\
\hline $\begin{array}{l}\text { Han desarrollado } \\
\text { mi capacidad para } \\
\text { resolver problemas } \\
\text { complejos. }\end{array}$ & & & & & \\
\hline
\end{tabular}

Marque el grado de acuerdo frente a las siguientes frases con respecto a $\underline{\mathbf{l a}}$ realización de resúmenes críticos de las lecturas.

\begin{tabular}{|l|l|l|l|l|l|}
\hline & $\begin{array}{c}\text { Muy de } \\
\text { acuerdo }\end{array}$ & De acuerdo & $\begin{array}{c}\text { Ni de } \\
\text { acuerdo } \\
\text { ni en } \\
\text { desacuerdo }\end{array}$ & En desacuerdo & $\begin{array}{c}\text { Muy en } \\
\text { desacuerdo }\end{array}$ \\
\hline $\begin{array}{l}\text { Han desarrollado } \\
\text { mi capacidad para } \\
\text { argumentar. }\end{array}$ & & & & & \\
\hline $\begin{array}{l}\text { Han desarrollado } \\
\text { mi capacidad para } \\
\text { plantear hipótesis. }\end{array}$ & & & & & \\
\hline $\begin{array}{l}\text { Han desarrollado } \\
\text { mi capacidad para } \\
\text { emitir juicios de } \\
\text { probabilidad. }\end{array}$ & & & & & \\
\hline $\begin{array}{l}\text { Han desarrollado mi } \\
\text { capacidad de decidir. }\end{array}$ & & & & & \\
\hline $\begin{array}{l}\text { Han desarrollado } \\
\text { mi capacidad para } \\
\text { resolver problemas } \\
\text { complejos. }\end{array}$ & & & & & \\
\hline
\end{tabular}




\section{Parte II: Responsabilidad Social}

Marque el grado de acuerdo frente a las siguientes frases con respecto a $\underline{\mathbf{l a}}$ totalidad de la experiencia de práctica en el curso

\begin{tabular}{|l|l|l|l|l|l|}
\hline & $\begin{array}{l}\text { Muy de } \\
\text { acuerdo }\end{array}$ & De acuerdo & $\begin{array}{c}\text { Ni de acuerdo ni } \\
\text { en desacuerdo }\end{array}$ & En desacuerdo & $\begin{array}{c}\text { Muy en } \\
\text { desacuerdo }\end{array}$ \\
\hline $\begin{array}{l}\text { Me ha permitido } \\
\text { reconocer la } \\
\text { importancia } \\
\text { de la } \\
\text { responsabilidad } \\
\text { social. }\end{array}$ & & & & & \\
\hline $\begin{array}{l}\text { Me ha permitido } \\
\text { reconocer las } \\
\text { capacidades que } \\
\text { poseo y pueden } \\
\text { ser útiles para la } \\
\text { responsabilidad } \\
\text { social. }\end{array}$ & & & & & \\
\hline $\begin{array}{l}\text { Me ha } \\
\text { incentivado a } \\
\text { participar de } \\
\text { proyectos de } \\
\text { responsabilidad } \\
\text { social. }\end{array}$ & & & & & \\
\hline
\end{tabular}

¿He participado de algún proyecto de responsabilidad social antes del presente semestre?

... No ... Si ¿Cuál?

¿Durante el semestre me he inscrito para participar de algún proyecto de responsabilidad social?

... No ... Si ¿Cuál?

¿Pienso inscribirme en algún proyecto de responsabilidad social en el futuro? ... No ... Si ¿Cuál?. 


\section{Anexo B}

\section{Rúbrica Pensamiento Crítico}

\begin{tabular}{|c|c|c|c|c|c|}
\hline & & \multicolumn{4}{|c|}{ Niveles de logro } \\
\hline & & 1 & 2 & 3 & 4 \\
\hline 1 & $\begin{array}{l}\text { Capacidad de } \\
\text { argumentar }\end{array}$ & $\begin{array}{l}\text { No es capaz } \\
\text { de reconocer } \\
\text { argumentos } \\
\text { del texto ni } \\
\text { plantear uno } \\
\text { propio. }\end{array}$ & $\begin{array}{l}\text { El estudiante } \\
\text { reconoce los } \\
\text { argumentos del } \\
\text { texto pero no } \\
\text { plantea ninguno } \\
\text { propio. }\end{array}$ & $\begin{array}{l}\text { El estudiante } \\
\text { plantea una } \\
\text { argumentación } \\
\text { personal pero } \\
\text { no reconoce el } \\
\text { argumento del } \\
\text { texto. }\end{array}$ & $\begin{array}{l}\text { El estudiante } \\
\text { reconoce } \\
\text { argumentos del } \\
\text { texto y plantea } \\
\text { argumentos } \\
\text { personales. }\end{array}$ \\
\hline 2 & $\begin{array}{l}\text { Capacidad } \\
\text { de plantear } \\
\text { hipótesis }\end{array}$ & $\begin{array}{l}\text { No se formula } \\
\text { ninguna } \\
\text { pregunta. }\end{array}$ & $\begin{array}{l}\text { Presenta una } \\
\text { hipótesis basada en } \\
\text { la intuición. }\end{array}$ & $\begin{array}{l}\text { Las hipótesis } \\
\text { recogen una } \\
\text { o más ideas } \\
\text { principales del } \\
\text { texto. }\end{array}$ & $\begin{array}{l}\text { Las hipótesis } \\
\text { son formuladas } \\
\text { de manera clara, } \\
\text { estructurada y } \\
\text { lógica. }\end{array}$ \\
\hline 3 & $\begin{array}{c}\text { Capacidad } \\
\text { de emitir } \\
\text { juicios de } \\
\text { probabilidad }\end{array}$ & $\begin{array}{l}\text { No se formula } \\
\text { ningún } \\
\text { juicio de } \\
\text { probabilidad. }\end{array}$ & $\begin{array}{l}\text { El juicio da cuenta } \\
\text { de un problema que } \\
\text { se puede discutir en } \\
\text { prácticas. }\end{array}$ & $\begin{array}{l}\text { El juicio es } \\
\text { idóneo para dar } \\
\text { cuenta de un } \\
\text { concepto. }\end{array}$ & $\begin{array}{l}\text { El juicio está } \\
\text { formulado de } \\
\text { manera clara, } \\
\text { estructurada y } \\
\text { lógica. }\end{array}$ \\
\hline 4 & $\begin{array}{c}\text { Capacidad de } \\
\text { decider }\end{array}$ & $\begin{array}{l}\text { No presenta } \\
\text { una postura } \\
\text { personal. }\end{array}$ & $\begin{array}{l}\text { Presenta una } \\
\text { postura personal } \\
\text { basada en la } \\
\text { intuición. }\end{array}$ & $\begin{array}{l}\text { Presenta una } \\
\text { postura personal } \\
\text { en base a una } \\
\text { discusión teórica, } \\
\text { un problema } \\
\text { practico o } \\
\text { en relación a } \\
\text { principios éticos. }\end{array}$ & $\begin{array}{l}\text { La postura } \\
\text { personal está } \\
\text { formulada de } \\
\text { manera clara, } \\
\text { estructurada y } \\
\text { lógica. }\end{array}$ \\
\hline 5 & $\begin{array}{l}\text { Capacidad } \\
\text { de resolver } \\
\text { problemas } \\
\text { complejos }\end{array}$ & $\begin{array}{l}\text { El estudiante } \\
\text { no plantea un } \\
\text { problema. }\end{array}$ & $\begin{array}{c}\text { El estudiante } \\
\text { plantea un } \\
\text { problema complejo } \\
\text { pero no plantea una } \\
\text { solución. }\end{array}$ & $\begin{array}{l}\text { El estudiante } \\
\text { plantea un } \\
\text { problema no } \\
\text { complejo y } \\
\text { plantea una } \\
\text { solución. }\end{array}$ & $\begin{array}{l}\text { El estudiante } \\
\text { plantea un } \\
\text { problema } \\
\text { complejo y una } \\
\text { solución. }\end{array}$ \\
\hline
\end{tabular}

